\title{
Author Correction: The chromosome-level quality genome provides insights into the evolution of the biosynthesis genes for aroma compounds of Osmanthus fragrans
}

Xiulian Yang ${ }^{1,2}$, Yuanzheng Yue ${ }^{1,2}$, Haiyan Li $i^{1,2}$, Wenjie Ding ${ }^{1,2}$, Gongwei Chen ${ }^{1,2}$, Tingting Shi ${ }^{1,2}$, Junhao Chen ${ }^{3}$, Min S. Park, Fei Chen ${ }^{3}$ and Lianggui Wang ${ }^{1,2}$

Correction to: Horticulture Research (2018) 5, 72; https://doi.org/10.1038/s41438-018-0108-0 published online 20 Nov 2018

Since the publication of this article, the authors have noticed that the NCBI accession number is missing from article.
NCBI accession number is: PRJNA529305

The authors would like to apologize for this error.

Published online: 19 April 2019

Correspondence: Min S. Park (minsungpark@grandomics.com) or 